\title{
Non-contact online thickness measurement system for metal films based on eddy current sensing with distance tracking technique
}

\author{
Wei Li, Hongbo Wang, and Zhihua Fenga) \\ Department of Precision Machinery and Precision Instrumentation, University of Science and Technology \\ of China, Hefei, Anhui 230026, China
}

(Received 28 January 2016; accepted 8 April 2016; published online 21 April 2016)

\begin{abstract}
This paper proposes an online, non-contact metal film thickness measurement system based on eddy current sensing. The slope of the lift-off curve (LOC) is used for characterizing target thickness. Theoretical derivation was conducted to prove that the slope is independent of the lift-off variation. In practice, the measurement has some immunity to the lift-off, but not perfect. The slope of LOC is still affected at some extent by the lift-off. Hence, a height tracking system was also proposed, which could stabilize the distance between the sensor and the target and significantly reduce the lift-off effect. The height tracking system contains a specially designed probe, which could vibrate rapidly to obtain a fast measurement speed, and its height can be adjusted up and down continuously to stabilize the lift-off. The sensor coil in the thickness measurement system was also used as the height sensor in the height tracking system. Several experiments were conducted to test the system performances under static and dynamic conditions. This measurement system demonstrated significant advantages, such as simple and clear conversion between the slope of LOC and target thickness, high resolution and stability, and minimized effect of lift-off variation. Published by AIP Publishing. [http://dx.doi.org/10.1063/1.4947234]
\end{abstract}

\section{INTRODUCTION}

Eddy current techniques are widely used in various technological and industrial applications, such as product quality testing, ${ }^{1-4}$ displacement and vibration measurement,${ }^{5-7}$ condition monitoring. ${ }^{8-15}$ With the many advantages, such as noncontact, inherently robust in harsh environment, insensitive to contaminants or any non-conductive material, simple to install and use, and low cost, eddy current techniques have been tremendously developed over the past few decades.

The requirements for the non-contact, highly precise online thickness measurement of metal films have rapidly increased with the development of industrial automation, semiconductor industry, and micromechanical technology. Researchers have proposed various non-contact methods for different measurement ranges, such as eddy current, ${ }^{9-17} \mathrm{X}$ ray reflectivity, ${ }^{18}$ and opto-acoustic ${ }^{19}$ techniques. For metal films with thickness from several to tens of nanometers, X-ray reflectivity and opto-acoustic techniques show good performances with high resolution and insensitivity to the material's properties. Eddy current technique allows increased flexibility and can cover a large measurement range from tens of nanometers to a number of millimeters. According to the types of excitation signals, the main techniques for metal thickness measurement with eddy current sensors can be classified as single-frequency eddy current, pulsed eddy current (PEC), and multi-frequency eddy current (MEC) sensors. The singlefrequency eddy current technique, ${ }^{9,12,14-16}$ where the coil is excited with a sinusoidal signal and the eddy currents are distributed in a fixed depth, is the most traditional method and

a)Electronic mail: fff@ustc.edu.cn is the basis of the other methods. The PEC technique, ${ }^{11,17}$ as a time-domain method, contains more frequency-rich information than the conventional single-frequency excitation. Three signal features, namely, the peak height, the time of occurrence of the first peak, and a characteristic zero-crossing time, were found to characterize the measurands, that is, thickness, conductivity, defect, and so on. For the frequency-domain method can provide information at different depths, MEC sensors were also developed to measure metal thickness or conductivity. ${ }^{10,13}$

For eddy current methods, an important factor limiting the response interpretation is the lift-off effect on the signals because the lift-off variation changes the mutual inductance between the excitation coil and the target. Reduction of the liftoff effect has been attempted with some successes by using the lift-off point of intersection (LOI), ${ }^{16,17,20}$ using multi-sensor coil, $^{2,21}$ and optimization of the sensor structure. LOI is an experimentally observed fixed point where the time-domain transient eddy current signals intersect as the lift-off solely changes. The LOI is immune to distance variation, thus the parameters of interest could be measured with high accuracy. However, complicated and advanced signal processing and interpretation are required to abstract the features of the signal and link the features explicitly to the sample conditions that are technically complex and expensive. For a large amount of sampling data, the computation is much greater than the time required for the measurements.

In our previous study, ${ }^{15}$ a method of measuring the thickness of metal films based on the immunity to lift-off variation was proposed. The lift-off curves, abbreviated as LOCs, of the sensor coil impedance in the L-R plane were shown as straight lines as the lift-off varies. In both finite element simulation and simple experiments, the slope of the LOC was verified as a 
good feature for characterizing the target thickness. However, some problems were observed. First, the theoretical analysis has not been developed perfectly. The hypothesis of the transformer model and the calculation of equivalent parameters are not precise. Second, the signal conditioning circuit could not modulate the resistance and inductance of the sensor coil accurately. As a result, the slope of LOC calculated by the output voltage was not proportional to the thickness. Third, the work pattern, in which the targets vibrated instead of the probe during the measurement, could not be applied in actual production. In this paper, the theoretical analysis was improved and the entire measurement system was established and tested. By optimizing the signal conditioning circuit, the slope of LOC exhibits a better linear relationship with the thickness. In practical application, the lift-off variation still resulted in measurement errors. In addition, a large lift-off reduced the sensitivity and the signal-to-noise ratio, whereas a small liftoff showed a risk of collision in the online production. Thus, this paper studied the effect of lift-off on thickness measurement and proposed a height tracking device to stabilize the lift-off distance. Without additional displacement sensors, the sensor coil was also used as the lift-off distance detector during the thickness measurement. Through closed-loop control, the probe tracked the vibrating target and worked with high sensitivity and precision. The static and dynamic performances of the system were tested in the simulated production environment. The results indicated that the measurement system has high resolution, strong stability, good reproducibility, and immunity to lift-off.

\section{PRINCIPLES OF THICKNESS MEASUREMENT}

A typical eddy current sensor consists of a sensor coil, a nearby target, and the signal conditioning circuit. When the sensor coil excited by the AC current approaches a conductive target, eddy currents are induced in the target. As a result, the coil resistance increases due to the nearby target, whereas the inductance decreases. The coil impedance is affected by several factors, such as lift-off, working frequency, the size of the target, and the electromagnetic properties of the target. The relationship among these factors is complicated; thus, we usually fix other physical quantities to measure parameters of interest.

\section{A. Theoretical support}

The sensor coil is the core component of the eddy current sensor. It is often considered as a resistance-inductance series circuit in the theoretical analysis. On the basis of conforming the design requirement, designers tend to use the sensor coil with more turns and drive the coil with higher excitation frequency. Because it is easier to measure the variation of large inductance with higher signal-to-noise ratio, and higher excitation frequency results in higher sensitivity. But more turns and layers lead to larger distributed capacitance of the sensor coil, which would be a fatal error source for high precision measurement of inductance especially when the excitation frequency is high. Thus, the structure of the coil and the number of turns should coordinate with the excitation frequency to make sure that the excitation frequency is much lower than the selfresonant frequency.

A simple transformer model, as shown in Fig. 1(a), was developed and widely used to analyze a few characteristics of eddy current sensors with effective results for nonferromagnetic targets. The primary circuit of the transformer represents the sensor coil, which has a serial resistor $R_{c}$ and an inductor $L_{c}$. $L_{t}$ (secondary inductance) and $Z_{t}$ (load impedance) are representative of the target and the coupling factor $M$ stands for the magnetic coupling between the sensor coil and the target.

$Z_{t}$ is complex functions of the geometric and physical properties of the target. The energy dissipation of eddy current in the target represents the resistance component. The distributed capacitance between the ring loops of eddy current represents the reactive component. According to the transmission line theory, the load impedance $Z_{t}$ is expressed as ${ }^{22}$

$$
Z_{t}=\frac{\sqrt{\frac{j \omega \mu_{0}}{\sigma}}}{\tanh \left(h \sqrt{j \omega \sigma \mu_{0}}\right)},
$$

where $\omega=2 \pi f$ is the angular frequency of the driving signal, $\sigma$ is the conductivity of the target, $\mu_{0}$ is the permeability of vacuum, and $h$ is the thickness of the target.

Here, the standard depth of penetration $\delta$ (skin depth) was introduced, which writes

$$
\delta=\sqrt{\frac{1}{\pi \mu_{0} \sigma f}} .
$$

The eddy current density in a conductor decreases exponentially from the value at the surface according to the depth from the surface. $\delta$ is defined as the depth below the surface of the conductor at which the current density has fallen to $1 / e$ of the surface current density. Considering that the target is ultra-thin $(h \ll \delta$ ), which corresponds to the working condition of the
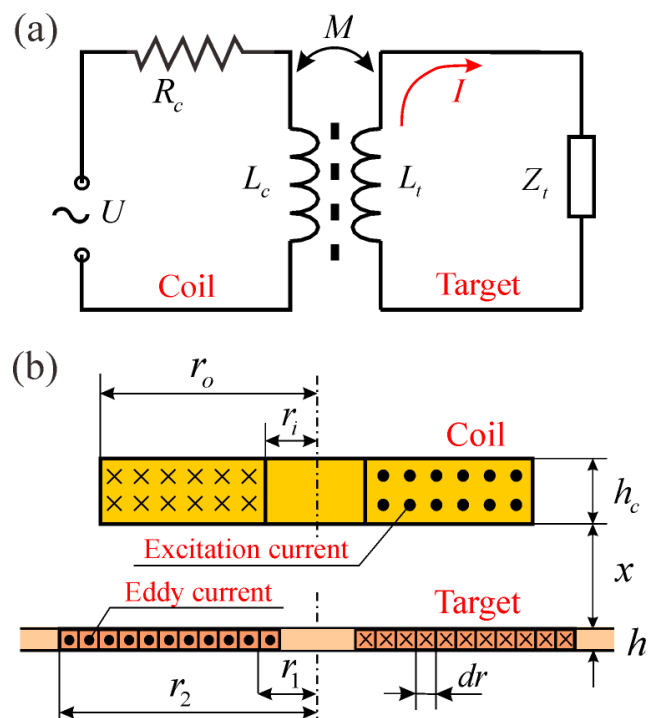

FIG. 1. (a) Transformer model when the excitation frequency is much lower than the self-resonant frequency; (b) geometry model for eddy current sensor. The target is thin metal film and the eddy current is uniformly distributed in the ring region. 
proposed method, a first order development of the hyperbolic tangent in Equation (1) allows to rewrite the $Z_{t}$ as

$$
Z_{t}=\frac{1}{h \sigma}=R_{t},
$$

which means that the load impedance is then purely resistive and does not depend on the frequency. So, for convenience, the load impedance $Z_{t}$ is replaced by the load resistance $R_{t}$ in the rest of this article.

The calculation of $R_{t}$ [Equation (3)] has no regard for the actual distribution range of the eddy current. When a hollow coil approaches to the target, most eddy current is induced in a ring-shaped region with inner and outer diameter of $r_{1}$ and $r_{2}$, as shown in Fig. 1(b). Both $r_{1}$ and $r_{2}$ are related to the geometric size of the probe $\left(r_{\mathrm{i}}\right.$ and $\left.r_{\mathrm{o}}\right)$, and they are almost constant as the distance $x$ changes in a proper range (Ref. 15, p. 4). Besides, the eddy current density on the target surface in the radial direction is almost the same. So, as shown in Fig. 1(b), the eddy current in the target is regarded as an array of ring loops and every ring loop has the same eddy current density $I_{d e n}$ with the unit $A / m$. Thus, the equivalent load resistant is a series connection of all the ring loop resistance and it can be calculated according to the same power consumption,

$$
\begin{gathered}
\int_{r_{1}}^{r_{2}}\left(I_{d e n} \cdot d r\right)^{2} \frac{2 \pi r}{\sigma h \cdot d r}=\left[I_{d e n} \cdot\left(r_{2}-r_{1}\right)\right]^{2} \cdot R_{t}, \\
R_{t}=\frac{\pi}{h \sigma} \cdot\left(1+\frac{2}{r_{2} / r_{1}-1}\right)=\frac{1}{h \sigma} \cdot S_{1}\left(r_{1}, r_{2}\right),
\end{gathered}
$$

where $S_{1}\left(r_{1}, r_{2}\right)$ is a function of $r_{1}$ and $r_{2}$. Equation (5) shows that the resistance of the target $R_{t}$ is in the inverse proportional relationship to the target thickness $h$, with no relation to the lift-off distance $x$ and the eddy current density $I_{d e n}$.

Based on the analytical solution of the disk coils, ${ }^{23}$ the equivalent inductance of ring loops $\left(h / r_{1} \ll 1\right.$ was assumed) was simplified as follows:

$$
L_{t}=\mu_{0} S_{2}\left(r_{1}, r_{2}\right) \text {, }
$$

where $S_{2}\left(r_{1}, r_{2}\right)$ is a function of $r_{1}$ and $r_{2}$.

According to Kirchhoff's law, the effective impedance $R_{s}$ and $L_{S}$ of the sensor coil (input impedance of the transformer) can be obtained as follows:

$$
\left\{\begin{array}{l}
R_{s}=R_{c}+\frac{\omega^{2} M^{2}}{R_{t}^{2}+\left(\omega L_{t}\right)^{2}} R_{t} \\
L_{s}=L_{c}-\frac{\omega^{2} M^{2}}{R_{t}^{2}+\left(\omega L_{t}\right)^{2}} L_{t}
\end{array}\right.
$$

where $R_{c}, L_{c}, R_{t}$, and $L_{t}$ are all constant if the sensor coil is chosen. If $\omega$ is a fixed angular frequency, the only variable in Equation (7) is the coupling factor $M$, which is the function of lift-off distance $x$. When the lift-off distance $x$ is changed, the coil resistance $R_{s}$ and inductance $L_{s}$ will change as well. When lift-off curve is drawn with the coil resistance $R_{s}$ as the horizontal coordinate and the coil inductance $L_{s}$ as the vertical coordinate, the slope of the lift-off curve $K$ is calculated by

$$
\begin{gathered}
L_{s}=-\frac{L_{t}}{R_{t}}\left(R_{s}-R_{c}\right)+L_{c}, \\
K=\left|-\frac{L_{t}}{R_{t}}\right|=\frac{\mu_{0} \sigma h}{\pi} \cdot \frac{S_{2}\left(r_{1}, r_{2}\right)}{S_{1}\left(r_{1}, r_{2}\right)},
\end{gathered}
$$

where $r_{1}$ and $r_{2}$ are treated as constants in the calculation and are only determined by the coil geometry $\left(r_{i}, r_{o}, h_{c}\right)$. Thus, for the non-ferromagnetic target, slope $K$ relies on three features, namely, the thickness of the target, the conductivity of the target, and the coil geometry. When a sensor coil is manufactured and the target material is known, the slope $K$ only depends on the thickness of the target.

\section{B. Implementation}

The key step of this method is how to produce the alternating change of the lift-off to get the LOC. For example, the sensor coil could be attached to a cantilever beam which is excited by a piezoelectric sheet. Each vibration period corresponds to an LOC, a slope of LOC, and a thickness result. Therefore, with a rigid cantilever, a fast measurement speed could be obtained. However, the probe size may be large, and an additional voltage amplifier is required.

Although the lift-off variation is used to measure the thickness in this method, an exceedingly large or small lift-off would not be a good working condition. A large lift-off reduces the sensitivity and the signal-to-noise ratio, whereas a small lift-off has a risk of collision in the online production. More important, to apply this method, the sensor coil must be working in the operating region where the LOC has a constant gradient, which requires the sensor coil not far from the target films. Therefore, a new motion probe, as shown in Fig. 2, was designed.

It has several design features. First, the probe could vibrate fast, up to $200 \mathrm{~Hz}$ with $50 \mu \mathrm{m}$ vibration amplitude. Thus, it allows fast measurement speed and fast target movement. Second, the sensor coil not only measures the target thickness but also works as a displacement sensor to measure the relative distance between the sensor coil and target. Third, through closed-loop control, the probe adjusts its height to track the moving target. So the lift-off maintains the proper distance during the measurement. Fourth, the tracking range can be changed according to different applications. The prototype in this paper has $2 \mathrm{~mm}$ tracking range. Structurally,

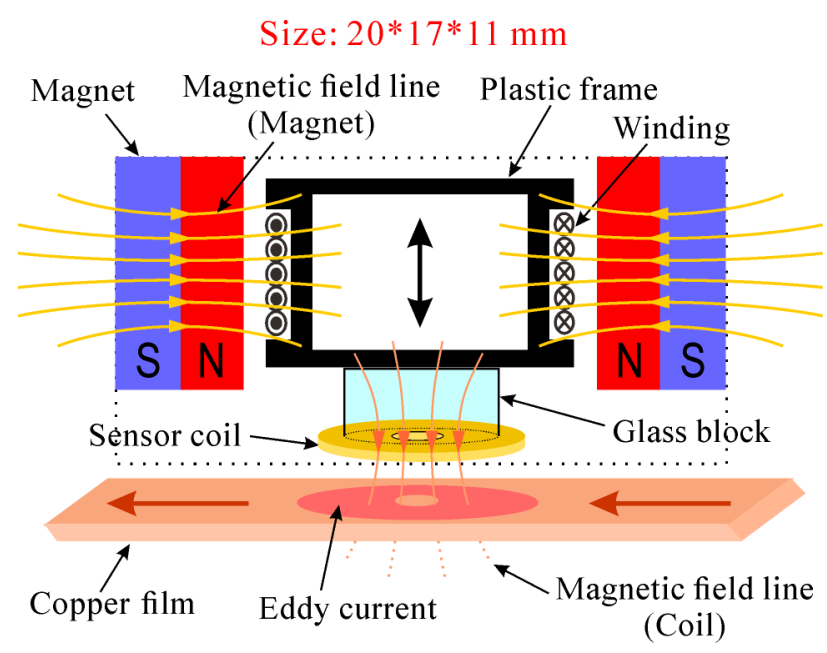

FIG. 2. Structure diagram of the sensor probe. The sensor coil is attached to the probe and it can vibrate at hundreds of Hertz to obtain the LOC. 
the sensor coil is attached to the plastic frame supported by four parallel steel wires. Different rigidity of the steel wires leads to different range of vibration frequencies. The winding around the frame is excited by the AC current to allow up and down vibration within the magnetic field of the magnets. The vibration frequency and amplitude are controlled by the frequency and amplitude of the AC current, while the average height of sensor coil is controlled by the DC bias of the AC current.

In the experiments, the resistance of the sensor coil was selected to reflect the lift-off. The basis of the selection is discussed in detail in Section IV. Using a closed-loop control, the probe could track the height of the target while vibrating at high frequency. The height tracking system is shown in Fig. 3(a). The vibration frequency of the probe was $110 \mathrm{~Hz}$. An up-down motion represents a cycle. $U_{R}$ and $U_{L}$ are the output voltage of the resistance and inductance, respectively, and are converted into discrete signals at the sampling rate of $11000 \mathrm{~Hz}$. The LOC was drawn with $U_{R}$ and $U_{L}$ [Fig. 3(b)], and the slope was calculated at every two cycles. An average slope was calculated and exported at every five slopes. $U_{R}$ was also used as the lift-off feedback information. An average $U_{R}$, called $R_{D C}$ [Fig. 3(b)], was calculated at every two cycles. $R_{D C}$ represented the average lift-off in two cycles. Using following feedback Equation (10), the offset of the control signal was calculated at every two cycles:

$$
\text { offset }[n]-\left(R_{D C}[n+1]-\text { level }\right) \cdot K_{C}=\text { offset }[n+1] \text {. }
$$

The parameter level is a reference voltage where $R_{D C}$ is controlled to be fixed during the tracking. It determines the lift-off distance directly when the height tracking system is working. In the experiment, level was set a value which corresponded to $800 \mu \mathrm{m}$ lift-off. $K_{C}$ is the conversion coefficient from the input voltage to the excitation voltage. When the height tracking system is used, the offset and the height of the probe change 55 times/s to track the target until $R_{D C}$ is fixed at the level.

In summary, the height tracking system plays two important roles in the measurement system. First, the height tracking system could stabilize the lift-off and further reduce the effect of the lift-off. Second, with the height tracking system, the measurement system could work at a more sensitive and safer condition than in former applications.

\section{DEVICE DESCRIPTION}

\section{A. Circuit}

The proposed eddy current sensor has a planar coil and a signal processing circuit to demodulate the resistance and the inductance signals. The sensor coil is wounded using a single crystallized copper wire that has a diameter of $50 \mu \mathrm{m}$ and consisting of 200 turns with outer and inner diameters of 7 and $1 \mathrm{~mm}$, respectively. As mentioned above, the excitation frequency of the coil must be far less than its self-resonant frequency. So an impedance analyzer was used to measure the coil inductance with the series model. With no target films around, the coil inductance with series model was measured under the excitation frequency from $10 \mathrm{kHz}$ to $1 \mathrm{MHz}$ (Fig. 4). The inductance is more stable below $400 \mathrm{kHz}$ because $400 \mathrm{kHz}$ is far less than the resonant frequency of the sensor coil. Considering the measurement range of the sensor, the excitation frequency was set at $100 \mathrm{kHz}$. The coil, including the connecting coaxial line, has an inductance of $129.4 \mu \mathrm{H}$ and a resistance of $25.5 \Omega$ at $100 \mathrm{kHz}$.

For the method proposed in this study, the film thickness is less than the penetration depth $(\delta)$. The penetration depth at the working frequency of $100 \mathrm{kHz}$ was calculated as $205 \mu \mathrm{m}$ using Equation (2). In the experiments, ten copper films with different thicknesses from $16 \mu \mathrm{m}$ to $151 \mu \mathrm{m}$ were used. The thickness of the copper films was measured using an electronic digital micrometer with the resolution of $1 \mu \mathrm{m}$. Each film was measured 20 times at different locations, and the mean value was calculated as the actual thickness. Because the target thickness is less than the penetration depth, the magnetic field of the sensor coil will pass through the target films with declining magnetic field strength, as shown in Fig. 2. So in actual application, other conductors should keep a distance with the target films.

In Section II, the LOC has been clarified as the key feature to measure target thickness. Thus, the accurate measurement of $R_{s}$ and $L_{s}$ of the LOC is the foundation of this method. For online measurement in industrial applications, an impedance analyzer connected to a computer is highly expensive and
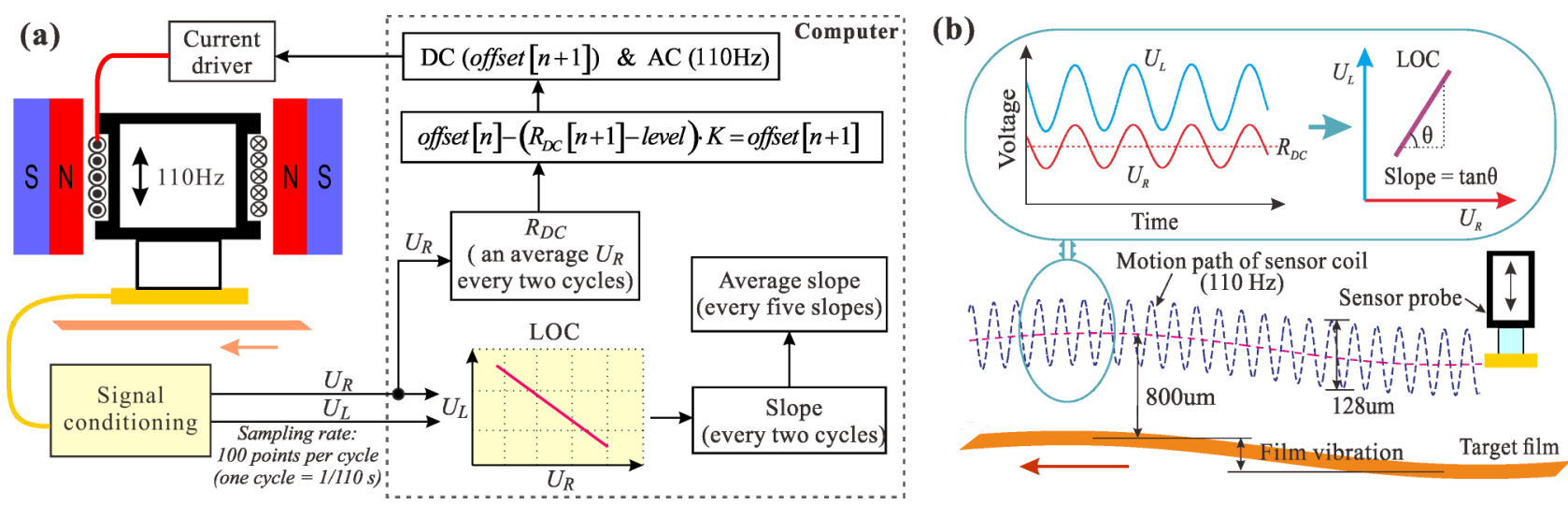

FIG. 3. (a) Program chart of LABVIEW: slope of LOC calculation and height tracking control; (b) the sensor probe tracks the slow vibration of target film while vibrating at high frequency $(110 \mathrm{~Hz})$. The lift-off is kept constant and $U_{R}$ and $U_{L}$ display stable waveform. 


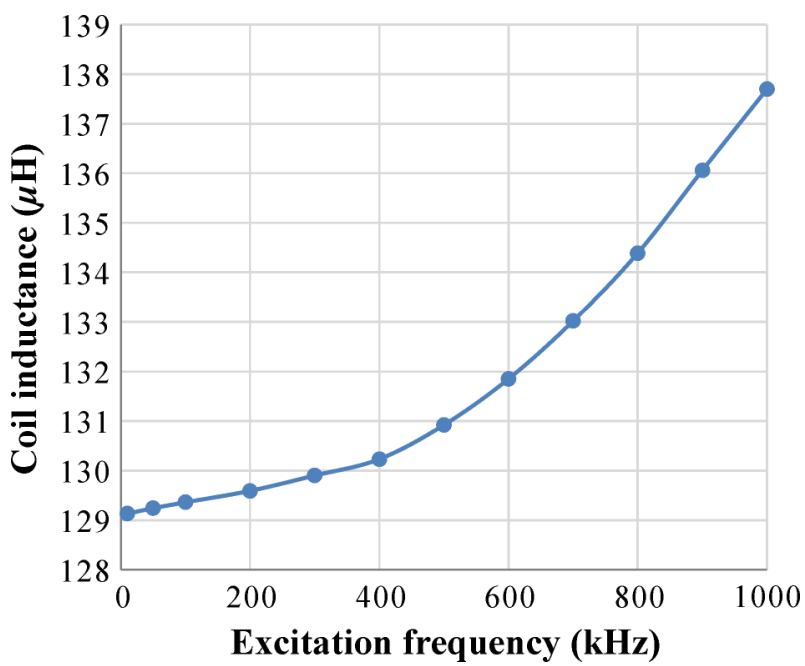

FIG. 4. The coil inductance measured by impedance analyzer with series model.

unsuitable, and the measurement speed is exceedingly slow. Therefore, a signal processing circuit, as shown in Fig. 5, is developed to demodulate the impedance of the sensor coil.

The circuit consists of a signal generator providing two orthogonal sine signal sources, a high-performance voltageto-current converter to drive the sensor coil and a dual-channel quadrature synchronous demodulator (lock-in amplifiers) to demodulate the resistance and inductance signals. In the online production, the lift-off distance changes largely, as well as the resistance and inductance of the sensor coil. The large variation of the coil impedance makes the $\mathrm{AC}$ bridge circuit ${ }^{7}$ unsuitable for the demodulation. A high-speed, wide-band, and large current output amplifier was selected to build the V-I convertor for driving the sensor coil. The tubular conducting shield of the coaxial line is connected to the inverting input of the amplifier, which has almost the same electric potential of ground. Thus, the conducting shield blocks most of the outside electromagnetic interference. However, when the sensor coil was connected as the feedback link of the amplifier, it provided high-gain to the high-frequency noise $(>100 \mathrm{kHz})$, which increased the interference of the circuit. To solve this problem, a capacitor $(1 \mathrm{nF})$ was connected in parallel with the sensor coil. The capacitance $1 \mathrm{nF}$ was selected not to largely affect the $100 \mathrm{kHz}$ signal and also to provide a low impedance path for the high-frequency noise. The output voltage of the amplifier is

$$
\tilde{V}_{i n}=\frac{R_{s}+j \omega L_{s}}{R_{4}} \tilde{e}(t),
$$

where $e(t)$ is the excitation voltage signal. Thus, the outputs of the processing circuit are

$$
\left\{\begin{array}{l}
U_{R}=\frac{2 e}{\pi R_{4}} \cdot K_{1} \cdot R_{S} \\
U_{L}=\frac{2 e}{\pi R_{4}} \cdot K_{2} \cdot \omega L_{s}
\end{array},\right.
$$

where $e$ is the amplitude of the excitation signal. Hence, the resistance and inductance are directly included in the two output channels.

\section{B. Movement device}

The sensor coil was attached to the movement device, as shown in Fig. 2. To decrease the effect of the magnets on the sensor coil, a glass block was used to increase the distance between them. The impedance of the sensor coil was measured with and without the magnets under the working frequency $100 \mathrm{kHz}$ and it changed less than a millesimal. The probe was very small, with a dimension of $20 \times 17 \times 10 \mathrm{~mm}$. The resonant frequency of the probe was around $90 \mathrm{~Hz}$ and it could be adjusted by changing the supporting wires. In the experiment, the vibration frequency of the probe was $110 \mathrm{~Hz}$.

\section{Experiment setup}

The experiment setup is shown in Fig. 6. The setup consists of the sensor probe and its signal-processing board, a moving copper target controlled by a stepper motor, an $\mathrm{AD} / \mathrm{DA}$ convertor board, and a computer to control and process the measurement and then displays the results. The moving target controlled by a stepper motor emulated the actual production environment.

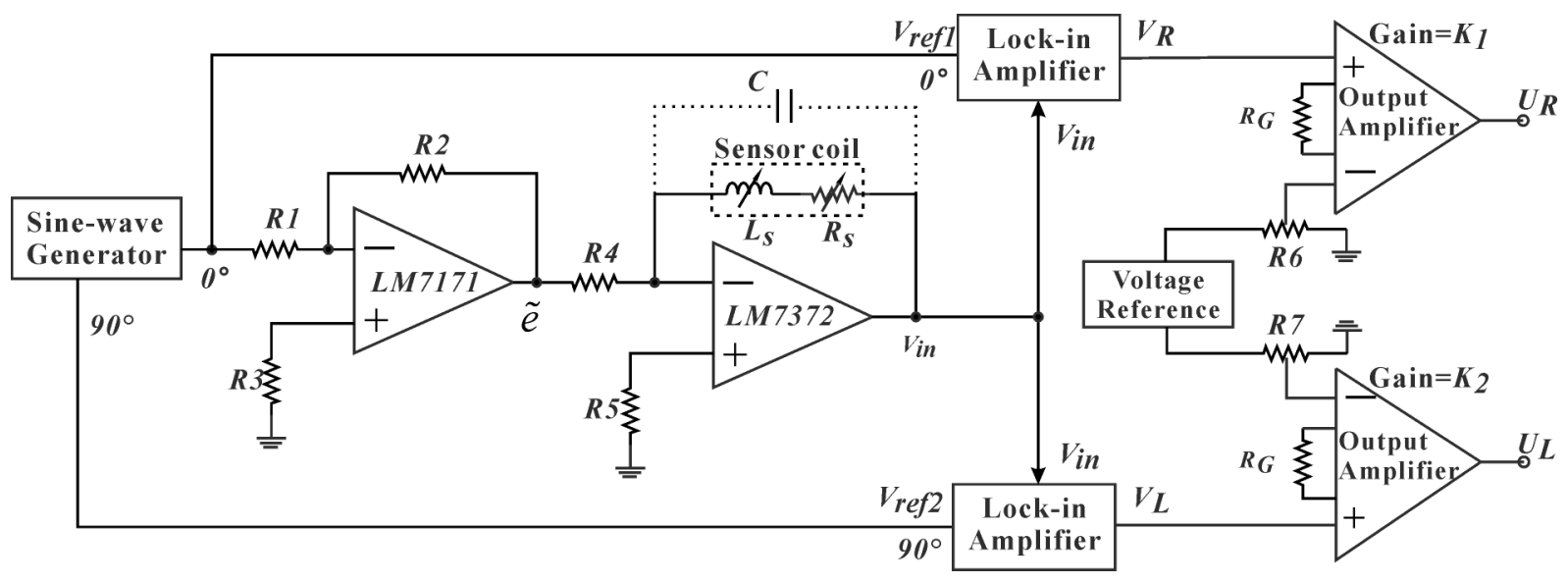

FIG. 5. Schematic of the signal processing circuit of the eddy current sensor. 


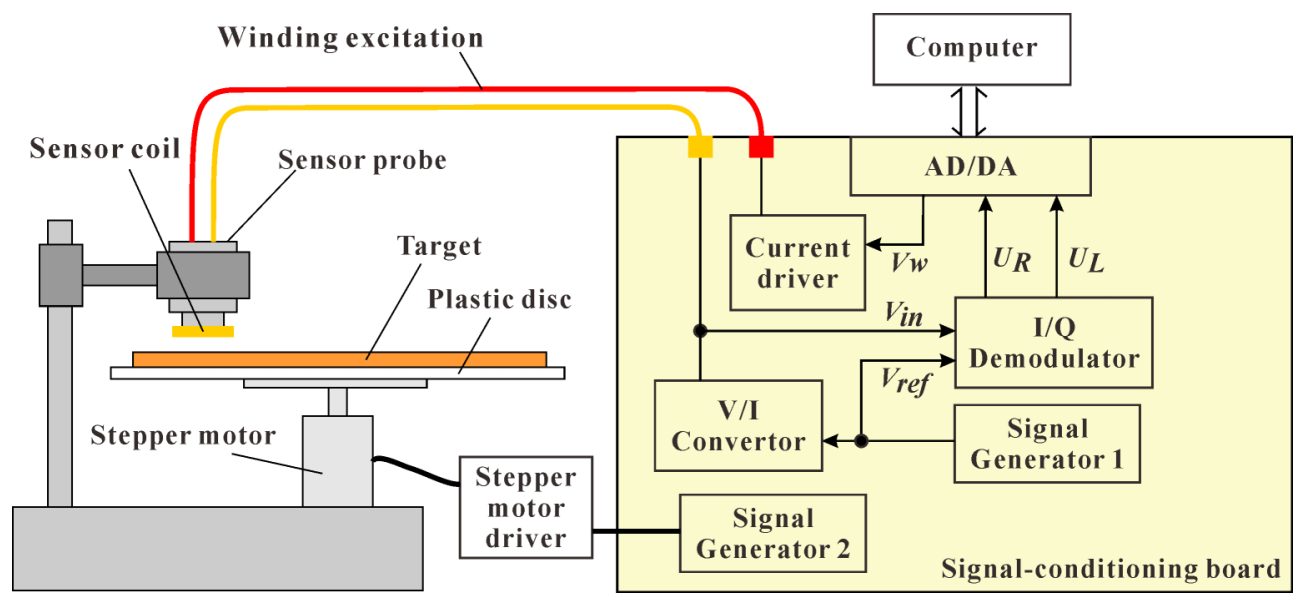

FIG. 6. The experiment setup emulating the actual production environment.

\section{EXPERIMENTS}

Three experiments were conducted to assess the performance of the system. First, the linear relation between the slope of LOC and the thickness was verified. Second, the height tracking plan was detailed and its effect was tested. Third, the overall performance of the system was tested with moving targets.

In the experiments, the excitation frequency of the sensor coil was $100 \mathrm{kHz}$, the vibration frequency of the probe was $110 \mathrm{~Hz}$, the vibration amplitude of the probe was $128 \mu \mathrm{m}$, and the circuit bandwidth was $500 \mathrm{~Hz}$.

\section{A. Linear relation between the slope of LOC and the thickness}

In this experiment, 10 copper films with thickness ranging from $16 \mu \mathrm{m}$ to $151 \mu \mathrm{m}$ were used. The target was fixed, and the height tracking system was not used here. 10 films were placed under the probe one by one, with the lift-off at $0.8 \mathrm{~mm}$. A good linear relation between the slope of LOC and the thickness is shown in Fig. 7(a). The system also displayed long-term stability in the measurement, as shown in Fig. 7(b). The slope of LOC was converted to thickness using the fitting formula in Fig. 7(a). Within the period of 3 minutes, for films with thickness of $16,34,60,84$, and $151 \mu \mathrm{m}$, the RMS noise was only $0.009 \mu \mathrm{m}(0.054 \%), 0.009 \mu \mathrm{m}(0.027 \%), 0.01 \mu \mathrm{m}(0.016 \%)$, $0.015 \mu \mathrm{m}(0.017 \%)$, and $0.021 \mu \mathrm{m}(0.014 \%)$, respectively. Thus, if this system measures a stationary target without target tracking, the resolution would be about $20 \mathrm{~nm}(0.02 \%)$.

\section{B. The height tracking plan}

The lift-off variation does not affect the slope in the derivation formula [Equation (9)] based on the transformer model, but the effect was observed in practice. For five films, when the lift-off changed from $0.5 \mathrm{~mm}$ to $1.1 \mathrm{~mm}$, measurement errors (without tracking) were observed in Fig. 8.

When the lift-off changed one tenth of the sensor coil diameter, a measurement error of $3.2 \%$ to $11.2 \%$ was observed. This result indicated that this method has some immunity to the lift-off, but not perfect. To achieve a higher request in industrial application, the height tracking system was designed to further reduce the effect of the lift-off. A height sensor was necessarily included in the tracking system to measure the lift-off and provide the feedback signal. To prevent the increase of system complexity, the sensor coil was also used as the height sensor. For traditional displacement sensors, the target thickness is much larger than the penetration
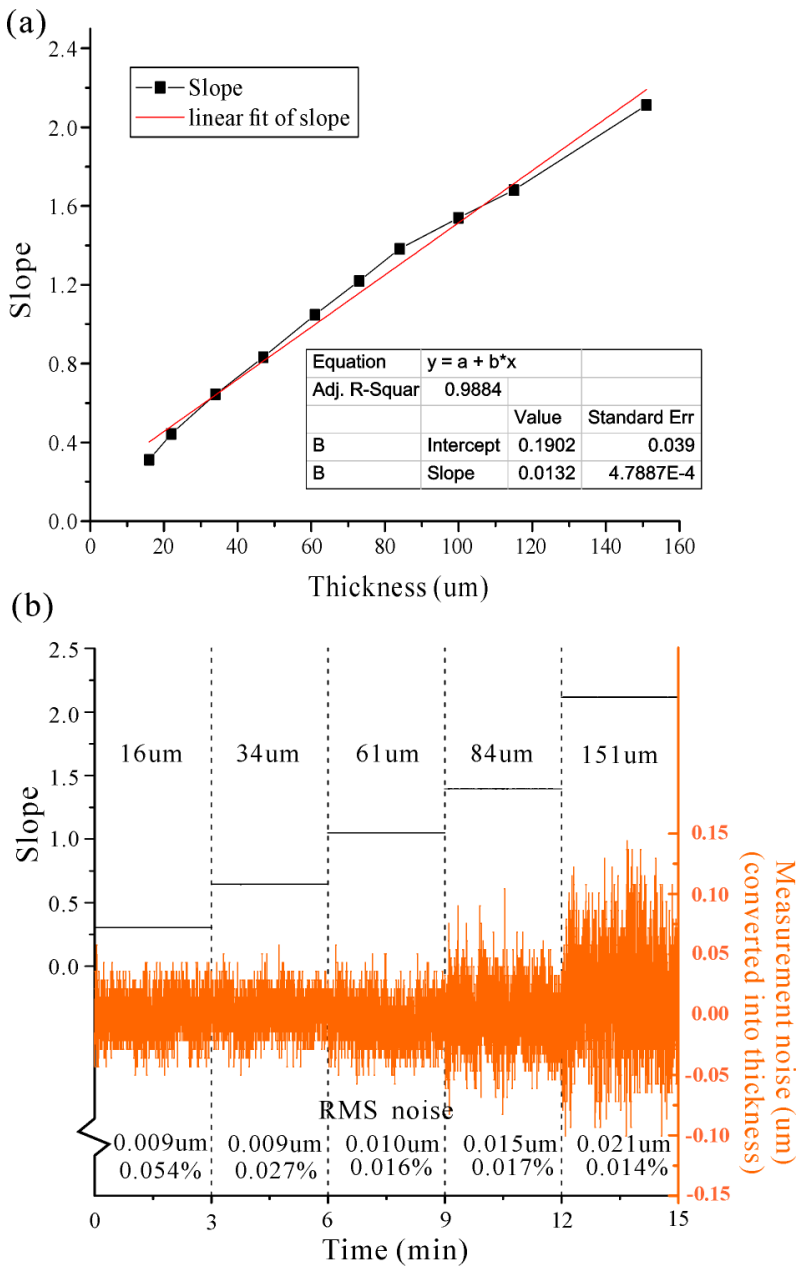

FIG. 7. (a) Linear relation between the slope of LOC and the target thickness; (b) long-term stability in the measurement. 


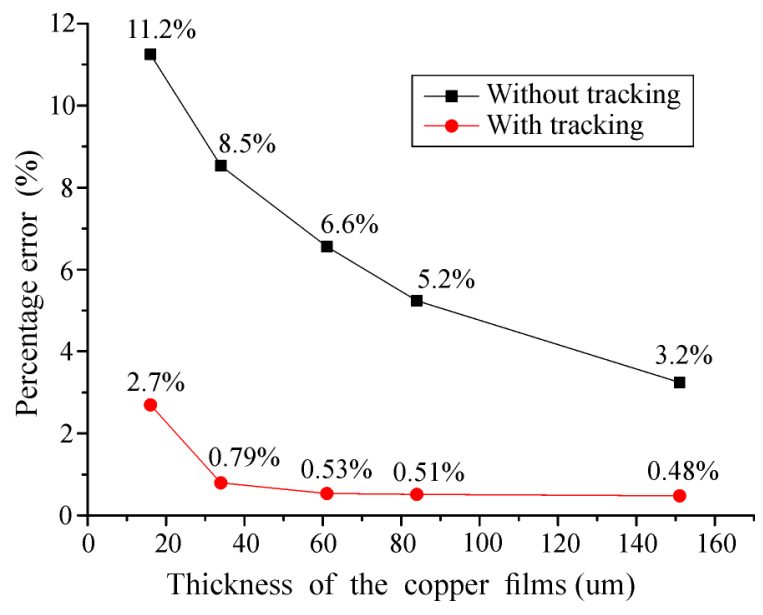

FIG. 8. Percentage of measurement errors when the lift-off changed from $0.5 \mathrm{~mm}$ to $1.1 \mathrm{~mm}$.

thickness. The equivalent resistance $R_{t}$ in Equation (7) is much smaller than $\omega L_{t}$, which means that the inductance $L_{s}$ has a better sensitivity to displacement. Thus, $L_{s}$ is commonly used to reflect displacement. However, in this method, the film thickness is less than the penetration thickness. In addition, the feature that reflects the height must be insensitive to the thickness variation. Therefore, two contrast tests were carried out to distinguish which parameter is more suitable for the height measurement: $L_{s}$ or $R_{s}$. One test showed the effect of thickness variation on the resistance and inductance outputs, while another test showed the effect of lift-off variation on the resistance and inductance outputs. The test results are shown in Fig. 9.

In the first test, the lift-off was fixed at $0.8 \mathrm{~mm}$, and the films with different thickness were replaced one by one. Figure 9(a) shows that the resistance output voltage has a gentler trend, and a maximum is observed at the thickness of around $40 \mu \mathrm{m}$. A finite element analysis (FEA) using COMSOL Multiphysics 4.4 was conducted, and a similar result as that in the experiment was obtained. The input resistance
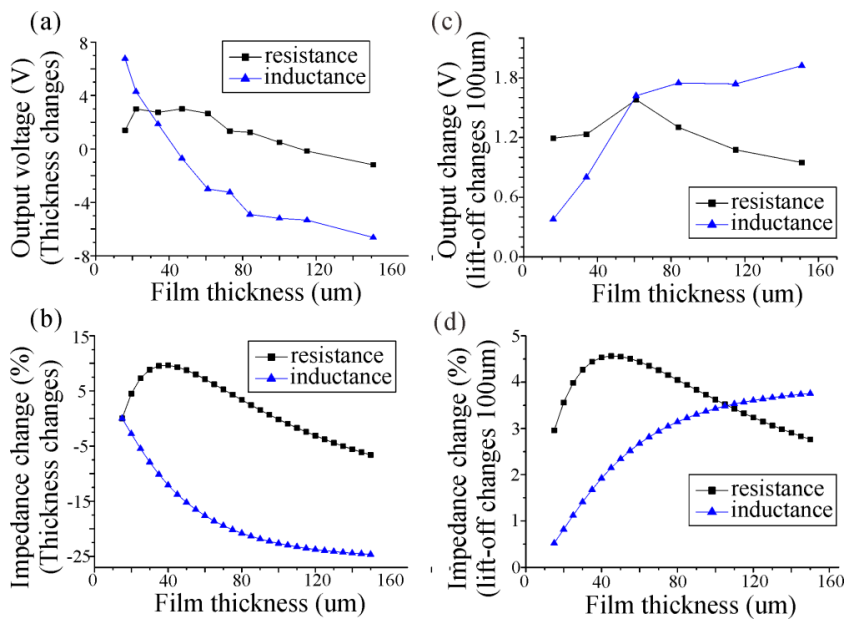

FIG. 9. Contrast tests to show that resistance is more suitable for the height measurement: (a) variation of the output voltage when the thickness changed; (b) results of the FEM to (a); (c) variation of the output voltage when the lift-off changed; and (d) results of the FEM to (c). of the sensor coil is strongly associated with the eddy current energy cost in the target. In exceedingly thin films, large equivalent resistance of the target impedes the eddy currents, resulting in a weak current and less energy cost. In sufficiently thick films, the equivalent resistance is small, and the eddy current intensity is constrained because of target resistivity and limited excitation. Therefore, the input resistance of the sensor coil has a maximum when the film is not too thin or too thick. In the second test, for films with different thickness, the sensitivity of $R_{S}$ and $L_{S}$ to lift-off variation was measured. The target films were moved up and down for $400 \mu \mathrm{m}$ to measure the change of the output voltage $U_{R}$ and $U_{L}$. The sensitivity of $U_{R}$ and $U_{L}$ to $100 \mu \mathrm{m}$ lift-off variation was calculated and the results were shown in Fig. 9(c). It can be seen that the resistance had a smaller fluctuation and a better sensitivity to the lift-off variation than inductance. The FEM results [Fig. 9(d)] also supported the experiment result. With the results of the two tests, the resistance of the sensor coil was selected to reflect the lift-off information for good sensitivity to the lift-off variation and immunity to the thickness variation. The peak to peak noise of the output voltage $U_{R}$ is less than $5 \mathrm{mV}$. The resolution of the lift-off measurement can easily achieve $1 \mu \mathrm{m}$. As shown in Fig. 3(a), the resistance voltage $U_{R}$ is the feedback signal which controls the offset of the excitation voltage.

After applying the height tracking system, the error caused by the lift-off variation has largely decreased [Fig. 8].
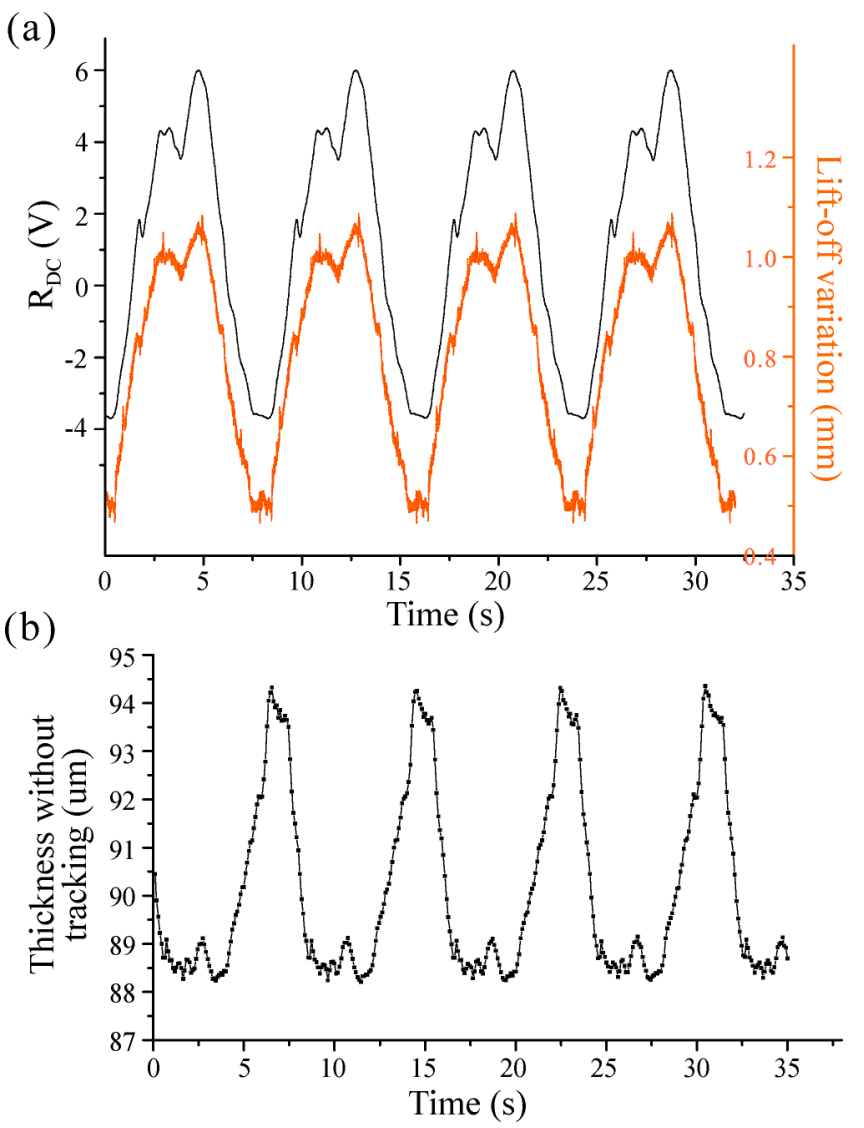

FIG. 10. Results without tracking: (a) the $R_{D C}$ curve well-matched the lift-off variation; (b) thickness measurement results. 

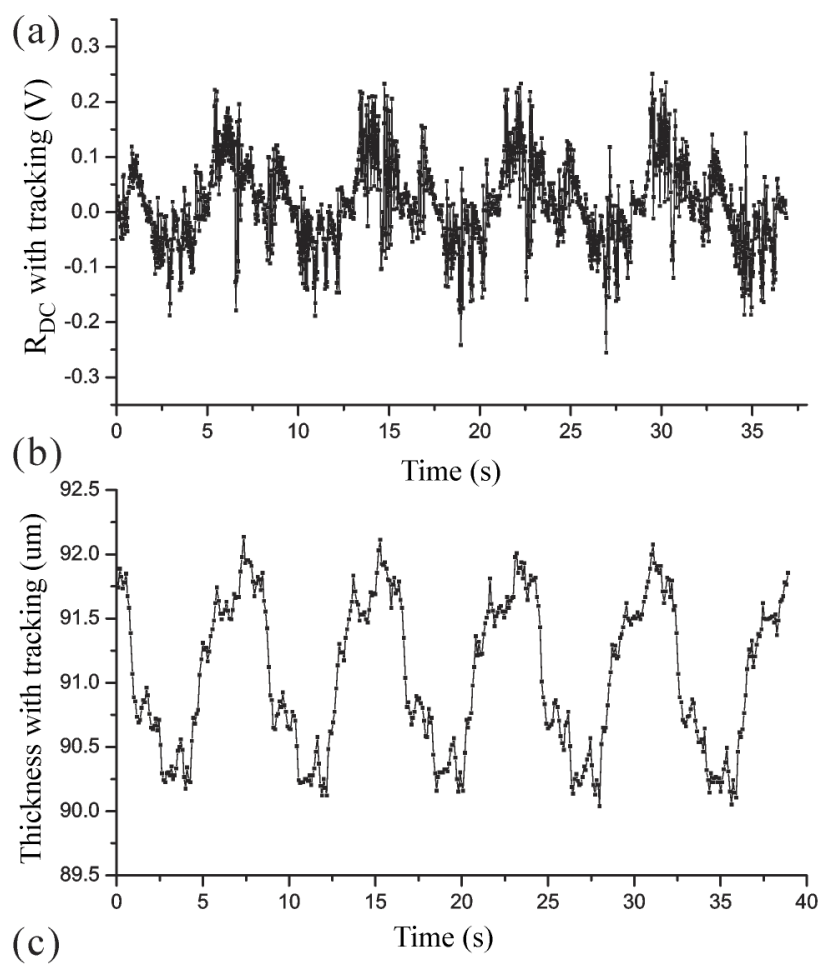

(c)

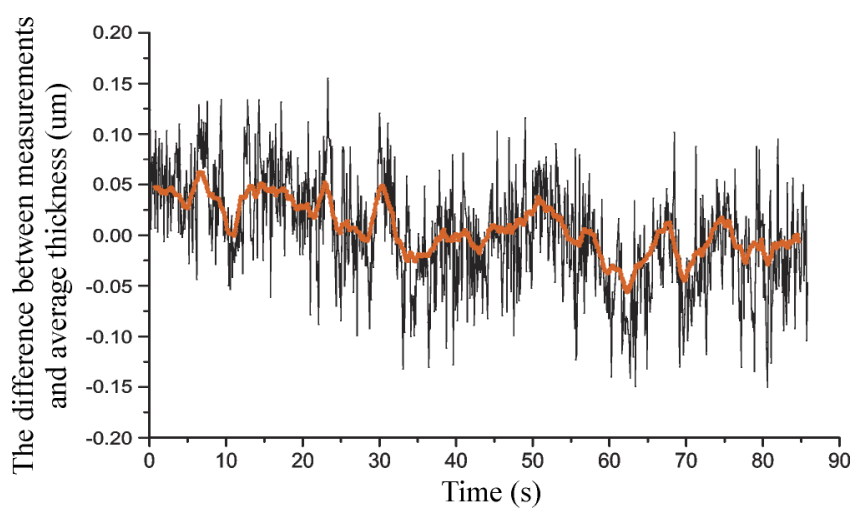

FIG. 11. Results with tracking: (a) small variation of $R_{D C}$; (b) thickness measurement results; and (c) the difference between measurements and average thickness.

\section{The overall performance}

To emulate the actual production condition, an $84 \mu \mathrm{m}$ thick copper film was glued to a plastic disc and rotated at $7.9 \mathrm{~s} /$ lap using a stepper motor. The probe was placed $40 \mathrm{~mm}$ from the disc center and the lift-off was at $0.8 \mathrm{~mm}$. Using a laser displacement sensor, the lift-off variation of the target under the probe was measured as $0.55 \mathrm{~mm}$ during the rotation. The target was tilted at about $0.8^{\circ}$; thus, the tilt of target film was ignored and only lift-off variation was taken into consideration. The lift-off variation and the $R_{D C}$ curve without height tracking are shown in Fig. 10(a). The two similar curves showed that $R_{D C}$ can reflect the lift-off. The measured thickness is shown in Fig. 10(b). Due to the calibration error between the fitted curve and the actual thickness, there was an error of about $7 \mu \mathrm{m}$ of the conversion. The fluctuation of the measured thickness contains two parts: thickness nonuniformity and lift-off variation.

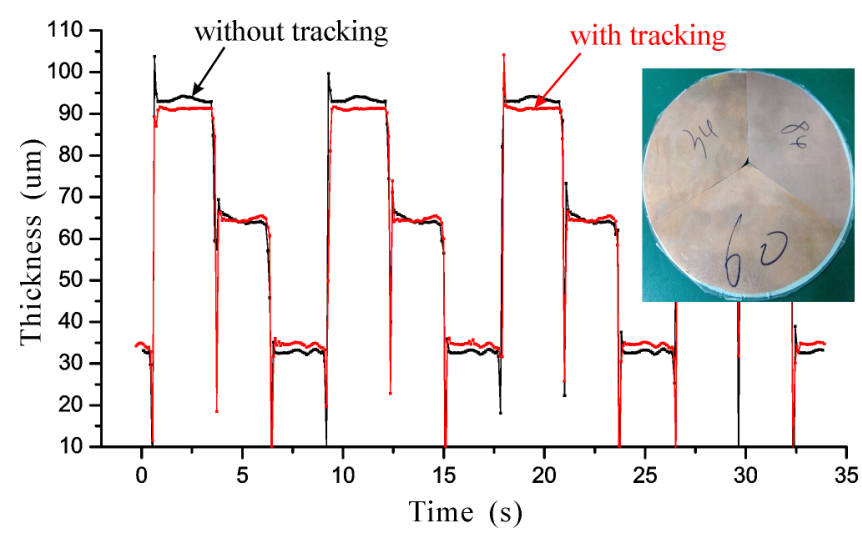

FIG. 12. Comparison between the measurement results, with and without tracking.

With height tracking, the variation of $R_{D C}$ was controlled within $0.4 \mathrm{~V}$ [Fig. 11(a)], which meant that the lift-off variation was only about $20 \mu \mathrm{m}(\ll 550 \mu \mathrm{m})$. Thickness measurement within a long time period $(87 \mathrm{~s}$ ) was conducted while the disc rotated 11 times. Fig. 11(b) shows the data in four cycles. The result shows the real thickness variation after eliminating the effect of lift-off variation. An average thickness was calculated using the measurements in 11 cycles. After subtracting the average thickness from the measurements, the difference [Fig. 11(c)] was within $\pm 0.1 \mu \mathrm{m}$ during $87 \mathrm{~s}$, which showed that the system had a good repeatability.

The next experiment measured three films with different thicknesses of 34, 60, and $84 \mu \mathrm{m}$. The disc rotated at $8.6 \mathrm{~s} / \mathrm{lap}$, and the lift-off variation of the target was $0.28 \mathrm{~mm}$. The measurement results and the picture of the disc are shown in Fig. 12. When the probe encountered the gaps between the films, a noise point with a very small thickness value was observed, which means that the measurement system has some ability to detect the target flaw in the online measurement. The with-tracking curve was smoother than without-tracking curve because it contained less interference of lift-off variation. And the thickness results were in excellent agreement with the measurements when the probe and the target stayed still.

\section{DISCUSSION AND CONCLUSION}

The system proposed in this study, which uses the slope of LOC feature of the eddy current sensor, has its unique advantages. First, the variation of the lift-off, which is a problem in other methods, was used in this study to obtain the slope of LOC and the thickness measurement. Using the height tracking system, the measurement is nearly immune to the liftoff. Second, the least-square fitting of the impedance signals to obtain the slope of LOC causes the measurement results to be insensitive to all minor data errors, from the measurement or other electromagnetic interferences. Third, the structure and parameters of the system could be changed according to different requirements. The probe is small and light, which means that the vibration frequency could be sufficiently high $(300 \mathrm{~Hz})$ to have a fast thickness measurement. For a highspeed target with small-amplitude vibration, the vibration frequency should increase appropriately. For a low-speed target 
with large-amplitude vibration, a larger sensor coil can be used. Meanwhile, reduce the vibration frequency of the probe and increase the excitation voltage of the winding to have a larger vibration amplitude. If the thickness of the target changes slowly, the thickness could be calculated with more resistance and inductance data. Fourth, the system accomplishes both thickness measurement and displacement measurement with only one sensor coil.

This method has two major contributions for the lift-off immunity. First, this measurement system has very small measurement error when the lift-off distance has a large change. With the height tracking system, the measurement error was reduced nearly $90 \%$ compared to the previous method (Fig. 9). Second, this method has an explicit and simple relation between the measurement result and the target thickness. Simple signal processing circuit and relatively high measurement speed make it a competitive method in the non-contact, online thickness measurement system.

This method is proposed only for the materials which are non-ferromagnetic and of high conductivity. The sensitivity of the sensor would be little different due to the difference of target conductivity. And the effective thickness measurement range is limited by the penetration depth $\delta$, which is related to the working frequency for a given material. High working frequency increases sensitivity but decreases the measurement range. Thus, the working frequency should be selected to meet the measurement range and then achieve high sensitivity. The proposed method can be used to measure thickness varying from tens of nanometers to several millimeters, with different driving frequencies.

In conclusion, this paper verifies the capability of the noncontact, online thickness measurement system. This system has significant advantages, such as having a simple sensor coil and signal processing, simple and clear conversion between slope of LOC and thickness, high resolution and stability, and minimized effect of lift-off variation by the tracking method. It provides a good approach for the non-contact, online metal films thickness measurement in advanced industrial applications, such as metal coating, production line of metal films, and applications in the semiconductor industry.

${ }^{1}$ J. Garcia-Martin, J. Gomez-Gil, and E. Vazquez-Sanchez, Sensors 11(3), 2525-2565 (2011).

${ }^{2}$ G. Y. Tian and A. Sophian, NDT\&E Int. 38(4), 319-324 (2005).

${ }^{3}$ N. Bowler and Y. Q. Huang, Meas. Sci. Technol. 16(11), 2193-2200 (2005).

${ }^{4}$ R. Romary, S. Jelassi, and J. F. Brudny, IEEE Trans. Ind. Electron. 57(1), 237-243 (2010).

${ }^{5}$ M. R. Nabavi and S. Nihtianov, IEEE Trans. Ind. Electron. 58(9), 4414-4423 (2011).

${ }^{6}$ M. R. Nabavi and S. N. Nihtianov, IEEE Sens. J. 12(12), 3346-3355 (2012).

${ }^{7}$ H. B. Wang and Z. H. Feng, Sens. Actuators, A 203, 362-368 (2013).

${ }^{8}$ W. Li, H. B. Wang, and Z. H. Feng, Rev. Sci. Instrum. 85(8), 085001 (2014).

${ }^{9}$ F. Sakran, M. Golosovsky, H. Goldberger, D. Davidov, and A. Frenkel, Appl. Phys. Lett. 78(11), 1634-1636 (2001).

${ }^{10}$ W. Yin and A. J. Peyton, NDT\&E Int. 40(1), 43-48 (2007).

${ }^{11}$ C. S. Angani, D. G. Park, G. D. Kim, C. G. Kim, and Y. M. Cheong, J. Appl. Phys. 107(9), 09E720 (2010).

${ }^{12}$ Z. L. Qu, Q. Zhao, Y. G. Meng, T. Q. Wang, D. W. Zhao, Y. W. Men, and X. C. Lu, Microelectron. Eng. 108, 66-70 (2013).

${ }^{13}$ E. Pinotti and E. Puppin, IEEE Trans. Instrum. Meas. 63(2), 479-484 (2014).

${ }^{14}$ Z. L. Qu, Q. Zhao, and Y. G. Meng, NDT\&E Int. 61, 53-57 (2014).

${ }^{15}$ H. B. Wang, W. Li, and Z. H. Feng, IEEE Trans. Instrum. Meas. 64(9), 2557-2564 (2015).

${ }^{16}$ A. L. Ribeiro, H. G. Ramos, and J. C. Arez, Measurement 45(9), 2246-2253 (2012).

${ }^{17}$ J. Kral, R. Smid, H. M. G. Ramos, and A. L. Ribeiro, IEEE Trans. Instrum. Meas. 62(7), 2043-2049 (2013).

${ }^{18}$ D. Windover, E. Barnet, J. Summers, C. Gribbin, T. M. Lu, A. Kumar, H. Bakhru, and S. L. Lee, AIP Conf. Proc. 550, 243-248 (2001).

${ }^{19}$ M. Gostein, T. C. Bailey, I. Emesh, A. C. Diebold, A. A. Maznev, M. Banet, M. Joffe, and R. Sacco, in Proceedings of the IEEE 2000 International Interconnect Technology Conference (IEEE, 2000), pp. $176-178$.

${ }^{20} \mathrm{C}$. Mandache and J. H. V. Lefebvre, NDT\&E Int. 39(1), 57-60 (2006).

${ }^{21}$ W. L. Yin and K. Xu, IEEE Trans. Instrum. Meas. 65(1), 164-169 (2016).

${ }^{22}$ Y. Le Bihan, NDT\&E Int. 36(5), 297-302 (2003).

${ }^{23}$ S. Babic and C. Akyel, IEEE Trans. Magn. 36(4), 1970-1975 (2000). 